\title{
Making Quality Improvement Happen in the Real World: Building Capability and Improving Multiple Projects at the Same Time
}

\author{
Malcolm Daniel, Alex Puxty, Barbara Miles \\ Glasgow Royal Infirmary, Scotland
}

\begin{abstract}
Improving work as part of clinical practice is challenging. Plans for improvement are often made, but not followed through.

A recent experience of failure in an ICU led to a change in approach. Members of the multi-professional team committed to meet weekly to learn about quality improvement by working on improvement projects.
\end{abstract}

The group selected four topics they wanted to work on. These were: a bundle for patients admitted with septic shock; early ( $\leq 4$ hours) sedation vacation after admission to ICU to allow titration of sedation to effect; achieving $\geq 20$ minutes of mobilisation per day in ventilated patients; and medicines reconciliation.

This quality improvement meeting was built into another regular weekly meeting. Initially the meeting ran for 30 minutes; each week some focused quality improvement teaching was provided in addition to talking about each individual project.

The team found the meeting useful, they saw the progress they were making but felt the allotted time was too short. After 6 weeks, the initial early results persuaded the team to increase the duration of this meeting to 45 minutes.

At the start reliability of each process was low (between $10 \%$ and $38 \%$ ). All four projects achieved their stated process reliability aim. This took between 165 and 334 days for each project. Many tests of change ideas were required to achieve this.

We have been able to improve multiple topics in a short period and produce sustainable change. The weekly meeting provided the focus to this improvement work. The teaching and coaching on quality improvement methodology that occurred as part of this meeting helped accelerate our rate of progress.

We believe this experience and the learning we have gained will help provide ideas for others who also want to improve healthcare delivery in different settings.

\section{Problem}

Like many other clinical units, the ICU at Glasgow Royal Infirmary (GRI) has always tried to improve the quality of care provided. In general the effects of these efforts were variable, sometimes things improved, sometimes things improved but only transiently, and at other times they remained unchanged.

The Scottish Patient Safety Programme changed that.[1] The application of improvement science principles on the discrete areas of activity contained within the critical care workstream produced larger effects than previously seen. The changes were also sustainable over time, and more importantly had effects on patient outcome.[2]

Two different doctors led the improvement work at GRI and at Stobhill Hospital. These hospitals merged their in-patient care in 2011. Each of these individuals had additional training in improvement science having taken part in the Scottish Patient Safety Programme (SPSP) Fellowship[3] over successive years.
Their colleagues were glad of this; it was good to have an individual to lead on this area and to take on the burden of this quality improvement work. After all it was not what many consider real science.[4,5]

The two doctors in the respective ICUs were reliant on their colleagues' participation in the SPSP work. Fortunately their colleagues were willing, if passive, participants. They were content for changes to be tried, but they did not have the time or the energy to learn more about improvement science.

The two doctors who led the work knew that to make wider improvements to care in the ICU they needed their multidisciplinary colleagues to develop these skills. Two people can only do so much. The two doctors knew their time would come; and it would be determined by the internal motivation of the rest of the team.

After the merger of GRI and Stobhill into one ICU on the GRI site in 2011, the charge nurses and consultants held an annual away day each January. They discussed and reviewed their work and specific 
areas of individual interest. They also made plans for what they would do in the year ahead. In January 2013 there was general agreement they wanted to improve the mobilisation of ventilated patients. The team strongly felt this would aid patient recovery and shorten time on a ventilator.

The team met again in January 2014. They reviewed what they planned and realised nothing had happened to improve mobilisation rates in ventilated patients. They were disappointed.

After a long discussion, the lead clinician exclaimed, "We should do more of this QI stuff, it's the only thing that makes a difference!". When asked what he thought we should apply "QI" to, he said "everything". The doctors with some additional improvement methodology expertise knew this was the time they had been waiting for, and that they had been presented with both a big opportunity and a big challenge in terms of the scale of the problem.

This article provides a description of how one ICU went about engaging their staff in quality improvement by working on four projects at one time. The ICU is large, there are 20 beds. The ICU has 98 whole time equivalent nursing staff, 12 consultants, and 8 trainees who rotate every three months.

The team chose to work on four projects initially. This provided the challenge to be able to lead multiple projects at the same time. We thought this would be helpful, as there would be learning from each one, and this learning would help accelerate the rate of progress of the other projects. The individual projects should be seen as part of an overall package. Each project had a process measure. In this report we describe how the reliability of each process improved, and also describe the changes made to improve the reliability of each process.

To improve the clinical outcomes and experiences of patients, clinical teams will usually have to work on multiple processes. We describe how we started and sustained a weekly quality improvement meeting. This was an important step to being able to improve multiple projects at the same time.

\section{Background}

The background to the problem this ICU faced is how do you create the time and space to provide some training on quality improvement methodology to a large number of staff who each may have different topics they want to improve? And do this while also providing a clinical service? This is a common problem that all clinical services face.

A recent report from the Health Foundation examined the evidence base for barriers to improvement in the NHS.[6] Many barriers were identified, among these were lack of time available, lack of confidence among professionals to make change, lack of improvement culture, and lack of perceived ownership of improvements.

\section{Baseline measurement}

The scale of the problem at the start of this project was clear. Over the 12-month period following the January 2013 meeting where the decision was made to improve mobilisation of ventilated patients next to nothing had happened.

On reflection some things were apparent. There was no clarity on what was to be achieved in terms of mobilisation, neither how mobilisation would be measured, nor were there any ideas about how to make mobilisation of ventilated patients happen more often. The mobilisation of ventilated patients did happen occasionally, but only when the consultant who was really interested in this was working in the ICU. This change had not been built into regular everyday practice. We measured reliability of the process in the first month of this work, it was $30 \%$.

The work described in this report also describes improving reliability of a septic shock bundle. Another consultant had been working on this for some time by collecting data. There were 13 months of data before the quality improvement meetings began. The results had varied on month-by-month basis; the median bundle reliability was $38 \%$.

There had been no previous measurement of reliability of early ( $\leq 4$ hours) sedation hold after admission to ICU; in the first month of this work it was $10 \%$. The initial reliability of medicines reconciliation was $25 \%$

\section{Design}

The ICU had a regular weekly grand round meeting on a Tuesday afternoon. The care of all patients in the ICU was discussed at this meeting. At the end of the away-day meeting the team resolved to start the weekly grand round meeting 15 minutes earlier than usual and finish it 15 minutes later. This would create 30 minutes each week for the team to come together talk about the progress made in projects they were interested in and receive coaching on quality improvement methodology. This 30 -minute period would be held at the start of the meeting.

At the first meeting the team selected four projects they wanted to work on. The projects selected reflected different personal interests of the group. The topics chosen were: a bundle for patients admitted with septic shock; early ( $\leq 4$ hours) sedation vacation after admission to ICU to allow titration to effect of sedation; achieving $\geq$ 20 minutes of mobilisation per day in ventilated patients; and medicines reconciliation.

Each week at the quality improvement meeting the progress of each project was reviewed. In addition some focused teaching on quality improvement was provided. The aim of this was to progressively build quality improvement skills in the members of the team as the work is done. The coaching would increase knowledge, and as this was integrated into the team's improvement work faster progress this would be made. Gaining early results is a key part of improvement work.

We also hoped that part of the benefit of working on multiple 
projects at the same time, that the learning gained from improving one process would be shared and help the other projects improve too. Importantly, while we worked on these four projects, we thought the progress made would draw more staff into working on other quality improvement projects.

The areas covered in these sessions included: developing a charter to start an improvement effort; operational definitions; how to construct a run chart; how to interpret a run chart;[7] how to develop change ideas; PDSA testing at small scale; principles underlying high reliability design; and systems thinking.

The first two weeks were focused on developing a charter for each project. A good charter provides a general description that answers the key question "What are we trying to accomplish?".[8] A simple acronym (ADEPT: Aim; Data; Evidence; Process \& PDSA; and Team) was taught to team members as it contained some of the key features needed at this stage. One of the authors had learned about this tool while at the International Forum on Quality and Safety in Healthcare in 2009.

Aim is the first part of this. Specifying clearly in a "how much by when" manner the level of reliability to be achieved. For three projects, early ( $\leq 4$ hours) sedation vacation after admission to ICU to allow titration to effect of sedation; achieving $\geq 20$ minutes of mobilisation per day in ventilated patients; and medicines reconciliation, the process reliability aimed for was $95 \%$. In the septic shock bundle one of the components was achieving a physiological endpoint and the team knew this was harder than simply completing a process. For the septic shock bundle an aim of $\geq 80 \%$ reliability was set.

Data is required for all improvement work. The hallmark of quality improvement is data plotted on a time series graph such as a run chart. Initial coaching focused on what constituted process and outcome measures, and establishing operational definitions for these. This also included how and where the data would be recorded, and when and by whom it would be collected by.

Evidence for there being something to work on was produced from initial weekly data; this showed baseline reliability was in the range $10-38 \%$. This is good, as it demonstrated a great opportunity for improvement. The team's aim was to improve processes to improve outcomes for patients.

Process got the team to think about how the work in the ICU was actually done. It made them think about where it would be possible to try smart changes to make the process more reliable, and to test them. For example, the septic shock bundle, and early sedation vacation were to be completed soon after ICU admission. This allowed the team to build change ideas into what was already done as part of routine care as a way of improving reliability.

Teamwork is the last part of the acronym. Each project had a team of doctors and nurses as well as a pharmacist and physiotherapist working and learning together as they made progress with the project.
This framework was used and written on a flip chart page for each project. The flip chart for each project was placed on the ICU seminar room wall. This provided a ready reference point to hold teams accountable to, and helped focus discussions at the weekly quality improvement meetings.

\section{Strategy}

The four projects fell into two broad categories; either a process that occurred at the immediate time of admission of a patient to the ICU (septic shock bundle and early ( $\leq 4$ hours) sedation vacation); or a process that was linked to the ward round and was part of daily work (mobilisation of ventilated patients and medicines reconciliation).

The weekly meeting began with a review of the reliability of each process measure in the previous week. At the start of this work overall reliability was low. This discussion provided a means to keep a focus on the four projects, and also covered factors that may be contributing to the low reliability. This provided a basis for identifying change ideas to test and see if they helped.

PDSA testing for processes occurring at admission:

We provide a brief synopsis of the testing carried out. The initial management of septic shock and the early sedation vacation happened at the time of ICU admission. This could be at any point in the day. Initial testing for each project focused on an updated admission goals sheet.

Our theory was this would act as a prompt and reminder about what was required and also it would act as an easy means of data collection. We found that the admission goals sheet was not always completed, and that sections were not clear to staff. These were updated, made more specific, and highlighted clearly.

We learned that despite this the team were not always clear about what needed to be done. Often the piece of paper the admission goals were written on went missing. This latter aspect also caused problems with data collection.

To address this the admission goals relating to management of septic shock and the early sedation hold were written on the whiteboard that was on the wall behind each bed space. Our theory was that placing the admission goals on a whiteboard rather than a sheet of paper would provide a clear visual prompt for all members of the team, and as importantly it would not be lost. Our experience from testing was that the various steps were not always completed within the planned time. We then added review times for each step. This helped ensure completion of each step.

An issue specific to the sedation hold was a reduction in reliability that appeared every three months. These reductions coincided with the medical rotation changeover when new trainees started in the ICU. This was addressed by specifically covering this in our local induction. In addition, it was included at the end of medical handover daily briefing. Similarly this was also included in the nurses' daily safety briefing. These additional changes really 
helped.

PDSA testing for processes linked to the daily ward round:

Testing specific to the daily mobilisation of ventilated patients focused initially on a prompt on a piece of paper listing suitability of patients for a daily dangle. A daily dangle involved getting the ventilated patient sitting on the edge of the bed with their legs dangling over the side. This provided clarity of the indications and contraindications to this level of mobilisation. The daily goals sheet that was already used in the ICU was updated to include an area for mobilisation goals together with recording whether or not the goal was achieved. This also provided an easy way for the data to be collected. Over time this area of the daily goals sheet was modified to make it easier to record the data. An additional change was how to improve communication between nurses, physiotherapists, and doctors about which patients were to be mobilised. We noticed the physiotherapists arrived in the unit at the time the doctors were completing the end of medical handover briefing. We established the practice of discussing with the physiotherapists which ventilated patients were suitable for mobilising, and then sharing this information with the charge nurses.

The medicines reconciliation team focused on gaining information on the barriers to completing the process. They developed and tested a new page to be included in Carevue (our electronic ICU record). This was easier to complete.

The sequence of these changes is annotated on each process measure run chart that accompanies this report (run charts 1 to 4 ).

\section{Results}

Each project had a run chart of the process measure reliability produced; this was updated and reviewed weekly. All projects showed considerable improvement, all eventually reached the stated aim for process reliability.

The following run charts display the achievements on improving process measures.

Run chart 1: bundle for patients admitted with septic shock

Run chart 2: early ( $\leq 4$ hours) sedation vacation after admission to ICU to allow titration of effect

Run chart 3: achieving $\geq 20$ minutes of mobilisation per day in ventilated patients

Run chart 4: admission medicines reconciliation for all patients both medications documented and plan made.

The run charts are annotated and display the sequence of changes to improve each process measure.

The introduction of the weekly meeting provided a solid foundation. The meeting was built onto the front end of an existing meeting to which medical staff were already committed. This ensured high attendance. It was not a new freestanding event that required major changes to a working week.

The four projects all made major progress. Using the IHI Project Assessment Scale (0 to 5.0; in 0.5 increments), all reached 4.0 (> $50 \%$ progress to project goals). Two projects reached 4.5 , having demonstrated sustained improvement over time.[9]

The team thought that improving sedation management and improving mobilisation of ventilated patients would reduce average number of ventilated days per month. There was a sustained downward shift in this outcome measure with eight data points below the median (Run chart 5).

See supplementary file: ds6972.pdf - "5 QI run charts"

\section{Lessons and limitations}

All four projects did achieve their stated aim of reliability. The projects that improved fastest and showed sustained change over time were medicines reconciliation, and daily mobilisation. At the start of this work the aim was improve each of the projects to the stated aim within 120 days. Medicines reconciliation took 165 days, and daily mobilisation took 252 days to achieve this level of reliability. These processes are high volume daytime activities.

In contrast, achieving high reliability with sedation vacation after admission and septic shock bundle was more challenging. It took 334 days to achieve $95 \%$ reliability for the sedation vacation within four hours of admission to ICU. It took 365 days to achieve $80 \%$ reliability for the septic shock bundle. These processes happen at time of ICU admission to ICU. The process can happen at any point across the 24 hour time period. This reduced opportunities for testing changes. The number of patients admitted each month with a diagnosis of septic shock was low, never exceeding 15 patients a month. This in part also accounts in part for the wide variation on a month-by-month basis; one event more or less makes a big change to percentage reliability in a small sample.

An important lesson from the work on improving reliability of early ( $\leq 4$ hours) sedation hold after admission to ICU came after 10 months. We noticed that every three months reliability of the process dropped. This may simply have reflected common cause variation, however, the timing coincided with the month of the medical trainees starting their rotation. Often, the trainees had worked in other hospitals where an early ( $\leq 4$ hours) sedation hold after admission to ICU was not done, especially if the admission was outside daytime hours. To address this, we included this as part of their induction to the ICU. Updating trainees on the ongoing quality improvement work in the ICU continues to be part of their induction to the ICU. With the frequency with which medical trainees rotate, this point is generalisable to many other sites.

We believed the admission sedation hold and the daily mobilisation of ventilated patients would reduce overall sedation and improve retention of strength in our patients. Over the duration of this improvement work, there has been a sustained downward shift in the monthly average duration of ventilation that would support this 
belief.

The selection of the specific figure of $\geq 20$ minutes of mobilisation per day in ventilated patients was part of a planned introduction of this process. Duration of mobilisation has been specified as a quality measure by the Faculty of Intensive Care Medicine in the Guidelines for Provision of Intensive Care Services document.[10] Now that sustained improvement has been achieved, we reset the aim from June 2015 to be $\geq 30$ minutes of mobilisation per day in ventilated patients. Once that is achieved we will move to 45 minutes per day.

The weekly meeting worked. The ICU team found this useful, but too short. The ICU team was enthused to see the progress made when displayed on run charts. After six weeks, the time allocated to the meeting was increased to 45 minutes per week; people committing to start the meeting a further 15 minutes earlier achieved this. This demonstrates the importance of achievement of small early results that helps build will of individuals to commit time to this work.

When the focus is on improving many projects, it is important to take steps to avoid "projectitis" and to have a strategy that guides the overall work. To that end, we have used the A Route Map to the 20:20 Vision for Health and Social Care[11] as our overall guide. The projects chosen in this phase of our work align to the three priority areas identified as quality ambitions in the 20:20 Vision. The three quality ambitions are safe, effective, and person centred care, underneath this are 12 priority areas for improvement. We ensured each of the projects chosen aligned to one of the priority areas for improvement identified in the report. This has been particularly useful for when senior leaders have visited our quality improvement meeting. They see clearly how our activities were connected to their wider responsibilities. As a consequence of developing this quality improvement meeting, we have transformed a policy document into action at the front-line. Most importantly we have produced meaningful results.

In IHI's Execution of Strategic Improvement Initiatives To Produce System-Level Results white paper[12] the essential elements for strategic improvement are described: Will; Ideas; and Execution. ICU members of staff chose the first topics to be worked upon. This built on their personal internal motivation, rather than any externally set process. This provided the initial will to fuel the projects. For this report we deliberately chose to report on how we got four improvement projects to work. To achieve system level aims, teams will usually need to work on a portfolio of projects and not just a single project.[12]

Improvement always requires ideas; simply providing care in the same way will only ever achieve the same results. Three of the projects chosen were about a single process; only one involved a bundle. Each project though did require change ideas to make the individual process or bundle more reliable. These are highlighted on the run charts in the form of annotations, illustrating when a change was made.

When the will to improve exists, and there are ideas on how to make something better, it is execution that enables the will and ideas to be converted into to meaningful action. The execution framework has three interdependent components.

Firstly, setting and aligning the work to system level aims. Our work aligning our improvement work to the quality ambitions in the 20:20 does this. Secondly, another important component of an execution strategy is developing quality improvement capability in staff. This is another priority area for improvement that is also identified in the NHS Scotland 20:20 vision document, workforce development. Although many staff in the NHS may have been "improving quality" for a long time, the discipline that is and that comes from "quality improvement" methodology enables larger results to be achieved, in a shorter time, that are more likely to be sustainable. We have avoided teaching quality improvement as a separate topic. All teaching is directly linked to the work of improving the projects that have been chosen. The third component is managing local improvement on a day-by-day, week-by-week basis. The weekly meeting we developed provided this. This provided not only oversight, but also importantly an opportunity to contribute ideas on what additional changes may be worth testing to improve a process. More importantly, review of weekly data gave staff an opportunity to see the progress being made; the benefit of seeing the success from early wins helped sustain this work and build will.

The work described in this paper does need to be interpreted in light of the following limitations. Firstly, the work undertaken in a clinical unit that had previous experience of quality improvement work. The existing of a national safety programme[1] provided both previous experience of this type of work, and also additional training in quality improvement for two of the individuals involved. The existence of this program may limit the generalisability of our findings to other healthcare environments without similar programs. Secondly, while we have previously written about the importance of content of a national programme providing context,[2] on this occasion the members of the clinical team selected the content to work on.

We firmly believe though the central principle of building quality improvement training around doing actual quality improvement work holds true in any context. Much of what we have described in this article is how we have built quality improvement into the microsystem within which we work.[13]

\section{Conclusion}

Our key aims were all met, each individual project met its stated level of process reliability. We were able to engage staff and improve four separate projects at the same time. Each one, however, took longer than 120 days to achieve this.

The difference from the previous year's experience on improving mobilisation in ventilated patients and what happened after the quality improvement meeting started could not be more dramatic. Indeed as the lead clinician had stated back in January 2014 "....this QI stuff it's the only thing that makes a difference!"

We have been able to improve multiple topics in a short period and 
produced sustainable change. Our starting point was to commit to have a weekly meeting about quality improvement. The meeting was kept short, just 30 minutes at the start. Healthcare staff are busy, and finding time for additional meetings is often difficult. The meetings purpose was to learn together how to improve multiple projects at the same time. The weekly meeting included a review of the most recent data available for each project. As the staff saw the progress being made, they decided to make the meeting 15 minutes longer. Coaching on quality improvement tools and techniques was provided at the time each project needed it.

Building quality improvement capability within staff is best done by working on an actual improvement project.[14] This work has built on colleagues internal motivation, and we have taken steps to align this work to the 20:20 Vision. We believe our experience and learning will help others wanting to improve healthcare delivery in different settings.

The real demonstration of the value of this work is when we asked staff what we should improve next that ideas came forward readily. Fourteen separate topics were suggested, and the next four improvement projects have been chosen. Each of these is led by member of staff who did not lead one of the projects described in this report. Our work has engaged our colleagues and changed our approach to improvement. Our aim now is improve these four new projects within 120 days, so we can move on an improve other important areas in the care we provide patients.

\section{References}

1 Scottish Patient Safety Programme; 2008. Available from: http://www.scottishpatientsafetyprogramme.scot.nhs.uk/about-us Accessed 8th January 2016.

2 Daniel M, Booth M, Ellis K, Maher S, Longmate A. Details behind the dots: How different intensive care units used common and contrasting methods to prevent ventilator associated pneumonia. BMJ Qual Improv Report. 2015;4:doi:10.1136 bmjquality.u207660.w3069.

3 Scottish Patient Safety Programme Fellowship; 2015. Available from: http://www.qihub.scot.nhs.uk/education-and-learning/scottishquality-and-safety-fellowship.aspx Accessed 8th January 2016.

4 Marshall M, Pronovost P, Dixon-Woods M. Promotion of improvement as a science. The Lancet. 2013;381:419-21

5 Marshall M, Mountford J. Developing a science of improvement. Journal of the Royal Society of Medicine. 2013;106:45-50

6 What's getting in the way? Barriers to improvement in the NHS. London: The Health Foundation; 2015 Available from: http://www.health.org.uk/publication/what\%E2\%80\%99s-gettingway-barriers-improvement-nhs Accessed: 28th March 2016

7 Perla R, Provost L, Murray S. The run chart: A simple analytical tool for learning from variation in healthcare processes. BMJ Quality and Safety. 2011;20:46-51
8 Langley GJ, Moen R, Nolan KM, Nolan TW, Norman CL, Provost LP. Using the Model for Improvement. IN: The Improvement Guide: a practical approach to enhancing organizational performance. 2nd ed. San Francisco, CA: Jossey-Bass; 2009. p. 89 - 108

9 Assessment Scale for Collaboratives. Cambridge, MA: Institute for Healthcare Improvement; 2004. Available from: http://www.ihi.org/resources/pages/tools/assessmentscaleforcollabo ratives.aspx?hidemsg=true Accessed 10th November 2015.

10 Guidelines for the Provision of Intensive Care Services. London: The Faculty of Intensive Care Medicine / The Intensive Care Society; 2015. 1st Edition: Available from: http://www.ficm.ac.uk/sites/default/files/GPICS\%20-\%20Ed.1\%20\% 282015\%29 0.pdf Accessed 18th January 2016.

11 A Route Map to the 2020 Vision for Health and Social Care. Edinburgh: Scottish Government Health Department; 2013. Available from: http://www.gov.scot/Resource/0042/00423188.pdf Accessed 18th January 2016.

12 Nolan T. Execution of Strategic Improvement Initiatives to Produce System-Level Results. Cambridge, MA: Institute for Healthcare Improvement 2007. Available from: http://www.ihi.org/resources/Pages/lHIWhitePapers/ExecutionofStra tegiclmprovementInitiativesWhitePaper.aspx Accessed 18th January 2016.

13 Nelson EC, Godfrey MM, Batalden PB, Berry SA, Bothe AE, Jr., McKinley KE, et al. Clinical microsystems, part 1. The building blocks of health systems. Joint Commission Journal on Quality \& Patient Safety. 2008;34:367-78

14 Langley GJ, Moen R, Nolan KM, Nolan TW, Norman CL, Provost LP. Developing Improvement Capability. In: The Improvement Guide: a practical approach to enhancing organizational performance 2nd ed. San Francisco, CA: Jossey-Bass; 2009. p. $331-47$

\section{Declaration of interests}

Malcolm Daniel and Barbara Miles completed Patient Safety Fellowships supported by the Scottish Patient Safety Programme. Malcolm Daniel was a Health Foundation Quality Improvement Fellow (2010-2011).

\section{Acknowledgements}

We would like to thank all the staff in GRI ICU for their help, support and encouragement. We would particularly like to highlight the contributions of Jenny Cuthill, Helen Devine, and Pamela MacTavish.

\section{Ethical approval}

We followed the guidance issued by NHS Great Glasgow and Clyde in 2008 "Process for Consideration and Approval of Requests to 


\section{BMJ Quality Improvement Reports}

Conduct Non-research Projects within NHS GG\&C". The guidance made clear this work did not require ethical approval. This work was exempt from ethical approval. 